\title{
RELATIONSHIPS BETWEEN THE NECK, THE TEMPERO-MANDIBULAR AND THE TEETH
}

\section{- by Franne Mallen}

\section{INTRODUCTION}

The relationship between the dental profession and the physiotherapy profession is traditionally restricted to the temperomandibular joint. The association is, however, much closer as the structures of the neck and the upper quarter are intimately related to the teeth and the tempero-mandibular joint (TMJ). Physiotherapists are largely concerned with rehabilitation but rehabilitation for many years was merely functional. Physiotherapists had little or no effect on the actual structure which was causing the symptoms and our role was mainly prophylactic with the use of modalities and exercises to try to improve or to create the best situation possible. Now, however, there are therapists who are specifically trained in the management of joint pathology. With specific techniques we are able to change the structure of the joint by having an effect on ligaments, capsules and the articular cartilage and thereby altering the joint dynamics and biomechanics.

We deal with the neurological, muscular and skeletal systems. It is the assessment of the neurological, muscular and skeletal structures that will establish the cause of the disorder. The objective is to find and treat the primary or dominant structure at fault and to be able to assess its effect on the other two structures. In other words, to find the cause of the disorder and then to assess and treat the secondary effects of the disorder. All the structures of the upper quarter are interrelated and each facet is important to the whole and needs to be integrated with knowledge, experience and skill.

All maxillo-facial structures depend on the cranium as the point of origin. Suprahyoid muscles are attached to the mandible, while infrahyoid muscles are attached to the shoulder girdle, the scapulae and the sternum.

The spine comprises three main functional units in the body:

- cranio-cervical and that is related to the synovial temperomandibular joint;

- cervico-thoracic and its synovial gleno-humeral joint;

- lumbo-sacral which has the hip joint as the synovial joint.

\section{Etfect of Posture}

"Mouth breathers" have a posteriorly rotated occiput, so with the head back the occlusal contacts are posterior, with the head forward the occlusal contacts are anterior and with the head in neutral the occlusal contacts will be even. So the aim of the physiotherapist is to create an even head on neck posture which will then, in turn, create an even cervico-thoracic and lumbar sacral alignment. Then we will be able to create a physiological state of rest and the muscles and the joint structures will be at rest.

In people with a "poking-chin" posture, the posterior muscles hold the head back ie., the cranium is rotated backwards on the upper part of the neck. The antagonists of the muscles of mastication are the posterior cranio-cervical muscles and therefore the neck is extended. When the posterior part of the neck is extended the mandible is pushed forward and the distance between the chin and the sternum increases. The levator scapulae are lengthened and the structures at the back of the neck are compressed creating an entrapment neuropathy. The nerves that are affected in this instance are among others, the great auricular nerve which supplies the tempero-mandibular joint and the occipital nerve which supplies the muscles of the posterior occiput creating occipital headaches.

\section{Treatment}

Treatment of such conditions, I believe, starts with the tempero-mandibular joint and consists of mobilisations for pain. Small oscillatory movements, gentle movements that do not reach the end of range are applied. These are used to reduce pain and improve the condition of the tissues. These mobilisations can be extended to increase range of movement as well. Another part of the treatment is the application of massage and myofascial release to the lateral pterygoid muscles and the masseters and various forms of electrotherapy to help to reduce the oedema and thickening of structures.

The second part of the treatment is to the upper cervical spine to correct the biomechanics of the cranio-cervical and upper cervical segments. Mobilisations are used to relieve painful joints as well as to stretch the stiff structures. Treatment of the thoracic spine, the lumbar spine and the sacral structures are also often necessary.

The third part is to teach exercises to maintain the correct position of the neck and will include a mobilisation type of exercise as well as a stabilisation type of exercise. It is only with the correction of the muscular and skeletal structures that we can achieve a neutral position and thus create a physiological state of rest. By so doing there will be proper retraction, opening of the mouth and lateral movements of the mandible.

The techniques employed are specific, skilled and subtle. When dentists put apparatus into the mouth all of the above are affected and there is a change in proprioceptive and mechano-receptors. There is often a compensation in posture which leads to neck pain. Conversely, many a neck pain and a headache has been relieved simply by a bite plate.

\section{Conclusion and Summary}

Changes in neck movement change the occlusal contacts and the mandibular dynamics. For these to be normal one needs a physiologicall state of rest of the head on the neck, the temperomandibular joints and of the neck on the body. This can be achieved by physiotherapists trained in manual therapy and with a sound knowledge of biomechanics and pathology.

\section{Recommended Reading}

1. Rocabado M. Relationship of the Tempero-mandibular Joint to Cervical Dysfunction. Proc 3rd IFOMT conf. Vail, Colorado 1977:103.

2. Maitland G D, Corrigan B. Practical Orthopaedic Medicine. Butterworth \& Co Ltd 1983

3. Maitland G D. Peripheral Manipulation. Second Ed. Butterworths 1977.

4. Bogduk N. Headaches and the Cervical Spine. Proceedings of the Cervical Spine and Headache Symposium. Brisbane 1981:1-17.

5. Bogduk N. Greater Occipital Neuralgia. Current Therapy in Neurological Surgery. Long D M, Marcel Dekker, Toronto 1985:175.

6. Jull G A. Headaches Associated with the Cervical Spine - a Clinical Review. Grieve G: Modern Manual Therapy of the Vertebral Column. Churchill Livingstone 1986:322.

7. Jull G A. Headaches of Cervical Origin. Grant R. Physiotherapy of the Cervical and Thoracic Spine. Churchill Livingstone.1988:11;195. 